\title{
Local Treatment With Electrochemotherapy of Superficial Angiosarcomas: Efficacy and Safety Results From a Multi-Institutional Retrospective Study
}

\author{
MICHELE GUIDA, MD, ${ }^{1}$ LUCA G. CAMPANA, MD, PhD, ${ }^{2,3} *$ PIETRO CURATOLO, ${ }^{M D},{ }^{4}$ \\ SABINO STRIPPOLI, MD, ${ }^{1}$ ANTONIO BONADIES, MD, ${ }^{5}$ GRETHA GRILZ, MD, ${ }^{6}$ CARLO CABULA, MD, ${ }^{7}$ \\ ROBERTA ROTUNNO, MD, ${ }^{4}$ STEFANIA BUCHER, $\mathrm{MD}^{5}$ NICOLA SOLARI, $\mathrm{MD}^{8}{ }^{8}$ \\ ANTONIO SANTORIELLO, MD, ${ }^{9}$ SARA VALPIONE, MD, ${ }^{3,10}$ AND CARLO R. ROSSI, MD ${ }^{2,3}$ \\ ${ }^{1}$ Medical Oncology Unit, Istituto dei Tumori, Bari, Italy \\ ${ }^{2}$ Veneto Institute of Oncology IOV-IRCCS, Padova, Italy \\ ${ }^{3}$ Department of Surgery, Oncology and Gastroenterology, University of Padova, Italy \\ ${ }^{4}$ Department of Dermatology and Plastic Surgery, La Sapienza University, Roma, Italy \\ ${ }^{5}$ Plastic Surgery Unit, San Gallicano Dermatologic Institute IRCCS IFO, Roma, Italy \\ ${ }^{6}$ Breast Surgery Unit, Città della salute e della scienza di Torino, Italy \\ ${ }^{7}$ Oncologic Surgery, Ospedale Oncologico A. Businco, Cagliari, Italy \\ ${ }^{8}$ Surgical Unit 1, San Martino IST-IRCCS, Genova, Italy \\ ${ }^{9}$ Dipartimento di chirurgia generale e specialistica II Università di Napoli, Italy \\ ${ }^{10}$ Medical Oncology, The Christie NHS Trust, Manchester, United Kingdom
}

Background: Angiosarcoma is an aggressive vascular neoplasm with a high propensity for local recurrence. Electrochemotherapy is an emerging skin-directed therapy, exerting prominent cytotoxic activity, and antivascular effects. Its efficacy in angiosarcoma has not been investigated. Methods: This multicenter retrospective analysis reviewed patients who underwent electrochemotherapy from 2007 to 2014 for superficial advanced angiosarcomas. Bleomycin was administered intravenously and delivered within tumors by means of percutaneously applied electric pulses, according to the European Standard Operating Procedures for Electrochemotherapy. Tumor assessment was performed using RECIST (version 1.1). Toxicity (CTCAE, v4.0) and local progression-free survival (LPFS) were also evaluated.

Results: Nineteen patients (13 with locally advanced and 6 with metastatic angiosarcomas) were treated. Tumor sites were: scalp ( $\mathrm{n}=5$ ), breast $(\mathrm{n}=8)$, other skin sites $(\mathrm{n}=3)$, and soft tissue $(\mathrm{n}=3)$. Target lesions $(\mathrm{n}=54)$ ranged in size from 1.5 to $2.5 \mathrm{~cm}($ median, $2 \mathrm{~cm})$. Treatment was well tolerated. After 2 months, an objective response was observed in 12/19 (63\%) patients, complete in 8 (42\%). One-year LPFS within treatment field was $68 \%$. Local symptom improvement included palliation of bleeding (5/19 patients) and pain relief (6/19 patients).

Conclusions: Electrochemotherapy may represent a new locoregional treatment for selected patients with superficial angiosarcomas.

J. Surg. Oncol. 2016;114:246-253. (C) 2016 Wiley Periodicals, Inc.

KeY WoRDS: hemangiosarcoma; vascular neoplasms; neoplasm recurrence; local; electrochemotherapy; bleomycin drug delivery systems

\section{INTRODUCTION}

Angiosarcomas represent a heterogeneous group of rare vascular malignancies, accounting for less than $2 \%$ of all soft tissue sarcomas [1]. They may occur at any anatomic site, most commonly head and neck skin and breast, often in the field of previous radiotherapy or chronic lymphedema [2]. Angiosarcomas usually present with highgrade histology and multifocal disease, with a tendency for local recurrence and distant metastases [3]. Generally, the patients with cutaneous angiosarcoma fare significantly better than those with deepseated tumors [4,5]. Nevertheless, among all superficial sarcomas, angiosarcoma shows a significantly lower local control (5-year local recurrence-free survival, 24.9\%) and survival (5-year overall survival, $25.4 \%$ ) compared with other histotypes [6]. Despite multidisciplinary approach and adherence to treatment guidelines are crucial for outcome of sarcomas [7], optimal management of angiosarcoma has not been defined yet, and relies on a combination of surgery, adjuvant radiation and, in selected cases, chemotherapy $[1,4,8]$. Due to the high incidence of disease multifocality and the complexity of primary site anatomy, the local recurrence rate reported in scalp angiosarcomas ranges from $44 \%$ to $100 \%$ and, in radiation-associated angiosarcomas, from $26 \%$ to $92 \%$ [8-15]. Also in the locally advanced and metastatic settings, despite the activity of doxorubicin- and paclitaxel-based regimens, patient outcome remains still unsatisfactory, progression free-survival spanning between 4 and 8 months [16]. Furthermore, most patients are older than 70 years [17] and the risk of treatment-related side effects may discourage aggressive treatment. As a result, there is an unmet need for treatment options in this field. Electrochemotherapy (ECT) enhances the cytotoxic effect of two otherwise poorly permeant agents, bleomycin and cisplatin, by means of short electric pulses, which transiently permeabilise tumor cell membrane. As a result, an

Michele Guida and Luca G. Campana contributed equally to this work and should be considered first author.

Conflict of interest: None.

*Correspondence to: Luca G. Campana, MD, PhD, Department of Surgery Oncology and Gastroenterology, University of Padova and Surgical Oncology Unit, Veneto Institute of Oncology IOV-IRCCS, Via Gattamelata 64, 35128 Padova, Italy. Fax: 0039-0498218349. E-mail: maximizing@ hotmail.com; luca.campana@ioveneto.it

Received 4 January 2016; Accepted 8 February 2016

DOI $10.1002 /$ jso. 24287

Published online 9 May 2016 in Wiley Online Library (wileyonlinelibrary.com). 
increased drug concentration can be achieved within tumors [18]. Moreover, ECT targets tumor blood flow by exploiting two different antivascular mechanisms $[19,20]$. The first is a transient vasoconstriction, which increases drug entrapment into tumor tissue; the second is a delayed disrupting action on endothelial cells leading to irrecoverable damage of tumor vessels and to a further decrease in tumor circulation. Thanks to these antivascular effects, ECT is particularly advantageous in patients with vascular neoplasms, such as Kaposi sarcoma, as well as in bleeding tumors, irrespective of histotype [20-22]. On these bases, it is conceivable that ECT might also be effective in other, more aggressive, vascular tumors. The aim of this study was to evaluate the activity and safety of ECT in patients with angiosarcoma.

\section{PATIENTS AND METHODS}

\section{Patient Selection}

This was a multi-institutional retrospective study, approved by the local institutional review boards of participating centers. Patients with locally advanced or metastatic angiosarcomas who underwent ECT from July 2007 to May 2014 at eight Italian hospitals (Veneto Institute of Oncology, Padova; Istituto Tumori Bari; La Sapienza University, Rome; San Gallicano Dermatologic Institute, Rome; Città della salute e della scienza, Torino; Ospedale Oncologico A. Businco, Cagliari; San Martino Hospital, Genova; II University of Naples) were identified. Their cases were reviewed and follow-up information was obtained from treating physicians. Patient data were acquired from medical, operative and pathology reports and stored in a dedicated electronic database. The diagnosis of angiosarcoma was confirmed by a dedicated pathologist. ECT was offered upon multidisciplinary agreement, if surgical resection was excluded and patients where not suitable for, or refractory to, radiotherapy or systemic treatment. Standard ECT selection criteria are detailed elsewhere [23]. In patients with concomitant visceral disease, the indication to ECT was given to patients with of symptomatic superficial tumors and when at least 3-month life expectancy was estimated. Each patient gave written informed consent and was treated according to Good Clinical Practice recommendations.

\section{Treatment}

ECT was performed according to the European Standard Operating Procedures for ECT (ESOPE) [23]. The procedure was carried out under general anaesthesia or mild general sedation, as appropriate. Bleomycin was administered intravenously as a bolus, at the dose of $15,000 \mathrm{IU} / \mathrm{m}^{2}$. After an interval of $8 \mathrm{~min}$, short ( 8 pulses of $100 \mu \mathrm{s}$ duration, at a repetition frequency of $5,000 \mathrm{~Hz}$ ), high-voltage (400-960 V) electric pulses were delivered to the tumor by means of a needle electrode. Electrode conformation was chosen according to the tumor characteristics. The electric pulses were generated by a square wave pulse generator (Cliniporator ${ }^{\mathrm{TM}}$, IGEA, Carpi, Italy) suitable for clinical electroporation. Pulse application lasted between 8 and $28 \mathrm{~min}$ after the infusion of chemotherapy. The indication to further ECT cycles included: (i) the achievement of partial response (PR) or stable disease (SD) after the first ECT, (ii) the occurrence of disease progression or recurrence within ECT field, or (iii) out-of-field progression.

\section{Tumor Response and Toxicity}

For each patient, we registered up to seven measurable tumors (target lesions). Before, and 1 and 2 months following ECT, their largest diameter was measured by mean of a caliper and their sum was calculated. Tumor response was evaluated by using modified Response Evaluation Criteria in Solid Tumours (RECIST) [24]. No confirmatory biopsies were performed in this study. Local and systemic toxicity were graded according to the Common Terminology Criteria for Adverse Events (CTCAE, v4.0). Pain level was assessed by a 10-point Verbal Numerical Rating Scale (VNRS; $0=$ no pain; $1-2=$ mild pain; $3-6=$ moderate pain; $7-10=$ severe pain).

\section{Statistical Analysis}

The main analysis was descriptive. Time to response was calculated from the date of the first ECT to the appearance of complete response (CR) or PR, whichever came first. To do between-group comparisons for binary parameters (i.e., tumor response, skin toxicity, and pain) we used contingency tables and Fisher's exact test. Local progression-free survival (LPFS) indicated the time from ECT to local recurrence or progression within ECT field. PFS was calculated from the date of ECT to disease progression at any site, whichever came first. Overall survival (OS) was calculated from the date of first ECT until patient diseasespecific death. Kaplan-Meier curves were generated to estimate LPFS and OS. Two sided $P$ values of 0.05 or less were deemed significant. Statistical analysis was performed using the SPSS software (Statistical Packages for Social Sciences, IBM, NY, U.S., version 17.0).

\section{RESULTS}

\section{Patient Demographics and Tumor Characteristics}

We retrieved 19 cases treated between July 2007 and May 2014. Patient characteristics and tumor features are presented in Tables I and II, respectively. Patients were 15 women $(79 \%)$ and four men $(21 \%)$, with a median age of 69 years (range, 66-76). Thirteen $(68 \%)$ patients had locoregional disease, while $6(32 \%)$ had synchronous distant metastases. Tumor location was as follows: skin $(n=8$ patients, five with scalp angiosarcoma), breast $(n=8$ patients, two with primary breast angiosarcoma), and soft tissue ( $\mathrm{n}=3$ patients) (Figs. 1-3). In 12 (63\%) patients, angiosarcoma developed within the field of previous radiotherapy (administered for a different malignancy). The median interval between radiotherapy and the diagnosis of angiosarcoma was 8 years (range, 7-18). Six patients $(32 \%)$ had a history of chronic lymphoedema. We registered a total of 54 target lesions at baseline (median 2/patient, range 2-4), with tumor size ranging from 1.5 to $3.5 \mathrm{~cm}$ (median, $2 \mathrm{~cm}$ ). In two patients with angiosarcoma of the scalp, further superficial metastases were present, but they were not included among target lesions due illdefined borders and lack of reliability in measurement. Fifty $(92 \%)$ tumors represented a local recurrence at the site of previous surgical resection. In these patients, the median local relapse-free interval was 3.5 months (range, 2-10); relapse-free interval was longer in patients with R0 than R1 resection, 5.5 months (range, 2-11) versus 2.8 months (range, 1-6), respectively. The median distant relapse-free survival following surgical resection was 6 months (range, 2-17).

\section{Treatment}

Seven $(37 \%)$ patients underwent ECT under general anaesthesia, whereas $12(63 \%)$ under mild general sedation. One patient was receiving concomitant beyond progression paclitaxel chemotherapy at the time of ECT with palliative intent, because of treatment option exhaustion. The median hospital stay was 2 days (range, 1-3). After an interval of at least 2 months after ECT, all patients received additional treatments, according to disease extension, general conditions and physician choice. In particular, 15 patients had no further ECT, two patients received a second ECT cycle and two patients received up to three ECT cycles; six patients $(32 \%)$ received systemic treatment, after a median interval of 8.1 months (range, 6.5-9.4), due to superficial disease progression not amenable with 
TABLE I. Patient Characteristics $(\mathbf{n}=19)$

\begin{tabular}{|c|c|c|c|}
\hline Characteristic & Pts & & $\%$ \\
\hline \multicolumn{4}{|l|}{ Sex } \\
\hline Female & 15 & & 79 \\
\hline Male & 4 & & 21 \\
\hline \multicolumn{4}{|l|}{ Age, years } \\
\hline Median & \multirow{2}{*}{\multicolumn{3}{|c|}{$\begin{array}{c}69 \\
66-76\end{array}$}} \\
\hline Range & & & \\
\hline \multicolumn{4}{|l|}{ Site of primary disease } \\
\hline Scalp & 5 & & 26 \\
\hline Breast $^{\mathrm{a}}$ & 8 & & 42 \\
\hline Skin, other sites & 3 & & 16 \\
\hline Soft tissue & 3 & & 16 \\
\hline \multicolumn{4}{|l|}{ AJCC stage at diagnosis } \\
\hline IB & 1 & & 5 \\
\hline IIA & 5 & & 26 \\
\hline IIB & 3 & & 16 \\
\hline III & 9 & & 48 \\
\hline IV & 1 & & 5 \\
\hline \multicolumn{4}{|l|}{ FNCLCC Grade } \\
\hline $1-2$ & 5 & & 26 \\
\hline 3 & 14 & & 74 \\
\hline \multicolumn{4}{|l|}{ Presentation } \\
\hline Primary & 6 & & 2 \\
\hline Secondary ${ }^{\mathrm{b}}$ & 13 & & 68 \\
\hline \multicolumn{4}{|l|}{ Disease stage at enrollment } \\
\hline Locally advanced disease & 13 & & 68 \\
\hline Metastatic disease & 6 & & 32 \\
\hline Lung & 3 & & 16 \\
\hline Liver & 1 & & 5 \\
\hline Soft tissue & 2 & & 11 \\
\hline \multicolumn{4}{|l|}{ Focality } \\
\hline Solitary & 7 & & 37 \\
\hline Multifocal & 12 & & 63 \\
\hline \multicolumn{4}{|l|}{ Specific clinical presentations } \\
\hline Irradiated field ${ }^{\mathrm{c}}$ & 12 & & 63 \\
\hline Chronic lymphoedema ${ }^{\mathrm{d}}$ & 6 & & 32 \\
\hline \multicolumn{4}{|l|}{ Previous treatments } \\
\hline $\mathrm{S}$ & 11 & & 58 \\
\hline $\mathrm{S}+\mathrm{CT}$ & 3 & & 16 \\
\hline $\mathrm{S}+\mathrm{RT}$ & 2 & & 11 \\
\hline $\mathrm{S}+\mathrm{RT}+\mathrm{CT}$ & 1 & & 5 \\
\hline RT & 2 & & 10 \\
\hline \multicolumn{4}{|l|}{ Radicality of resection $(n=17)$} \\
\hline R0 & 11 & & 65 \\
\hline $\mathrm{R} 1$ & 6 & & 35 \\
\hline
\end{tabular}

$\mathrm{S}$, surgery; CT, chemotherapy; RT, radiotherapy.

${ }^{\text {a }}$ Two patients had primary angiosarcoma of the breast.

${ }^{b}$ Primary tumors included: breast cancer ( $\mathrm{n}=9$ patients), squamous cell carcinoma ( $\mathrm{n}=2$ patients), colorectal cancer $(\mathrm{n}=1$ patients), melanoma $(\mathrm{n}=1$ patient).

${ }^{\mathrm{c}}$ The median interval time from RT was 8.5 years (range, 7-10.8).

${ }^{\mathrm{d}}$ Location of lymphoedema was: trunk ( $\mathrm{n}=3$ patients) and limbs $(\mathrm{n}=3$ patients).

additional ECT or occurrence of distant metastases. Systemic therapies included gemcitabine $(n=3$ patients $)$, docetaxel $(n=1$ patient), and pazopanib ( $\mathrm{n}=2$ patients). At the last follow-up, five of these six patients showed disease progression.

\section{Local Tumor Response}

Two months after ECT, an objective response was observed in 47 $(85.5 \%)$ of 54 target lesions, including $36(66.7 \%)$ with CR and 10 $(18.5 \%)$ with PR. Five tumors $(9.3 \%)$ had SD and $3(5.6 \%)$ showed progressive disease (PD). As a result, 12 out of 19 patients $(63 \%)$ were responsive to treatment: $8(42 \%)$ reported CR, $4(21 \%) \mathrm{PR}, 6(32 \%) \mathrm{SD}$, and $1(5 \%)$ PD (Fig. 1-3).

In five of seven patients who initially achieved SD, we subsequently observed a PR, after a median interval of 3 months (range, 3-7) (Fig. 4). One of these five patients finally achieved local CR at 6 months while receiving systemic treatment with docetaxel, which was started
TABLE II. Tumor Characteristics and Outcomes

\begin{tabular}{|c|c|c|c|}
\hline Characteristic & No. & & $\%$ \\
\hline No. of treated tumors (target lesions) & 54 & & 100 \\
\hline \multicolumn{4}{|l|}{ No. per patient } \\
\hline Median & \multicolumn{3}{|c|}{2} \\
\hline Range & \multicolumn{3}{|c|}{$1-4$} \\
\hline \multicolumn{4}{|l|}{ Anatomical site } \\
\hline Scalp & 5 & & 9 \\
\hline Upper limb & 7 & & 13 \\
\hline Chest wall & 38 & & 70 \\
\hline Abdominal wall & 4 & & 8 \\
\hline \multicolumn{4}{|l|}{ Size, $\mathrm{cm}$} \\
\hline Median & \multirow{2}{*}{\multicolumn{3}{|c|}{$\begin{array}{c}2 \\
1.5-3.5\end{array}$}} \\
\hline Range & & & \\
\hline \multicolumn{4}{|l|}{ Response to ECT } \\
\hline CR & 43 & & 80 \\
\hline PR & 7 & & 13 \\
\hline SD & 4 & & 7 \\
\hline \multicolumn{4}{|l|}{ Time to response, days } \\
\hline Median & \multirow{2}{*}{\multicolumn{3}{|c|}{$\begin{array}{c}31 \\
28-41\end{array}$}} \\
\hline Range & & & \\
\hline \multicolumn{4}{|l|}{ Response duration, months } \\
\hline Median & \multirow{2}{*}{\multicolumn{3}{|c|}{$\begin{array}{c}6.5 \\
4.3-15.3\end{array}$}} \\
\hline Range & & & \\
\hline
\end{tabular}

ECT, electrochemotherapy; CR, complete response; PR, partial response; SD, stable disease; LPFS, local progression-free survival.

3 months after ECT. The outcome of the remaining two patients who initially had SD was as follows: one patient experienced disease progression 2.2 months after treatment, the other one maintained SD until his death, which occurred 7 months after ECT (Fig. 4).

Overall, in the 17 responsive patients, tumor regression was observed after a median interval of 31 days (range, 28-210 days) from the first ECT cycle.

In the five patients who were previously irradiated for angiosarcoma, local tumor response to ECT was as follows: CR in two cases, PR in two cases, no response in the patient who was receiving concomitant systemic treatment with paclitaxel.

The outcome of patients $(n=3)$ with soft tissue angiosarcomas was as follows: one patient achieved CR, and two patients PR (Fig. 4). The first patient, who was treated for a soft tissue angiosarcoma of the shoulder, remained local disease-free for over 2 years, until his death. The second patient achieved PR which was subsequently consolidated by the administration of further systemic treatment, thus leading to CR; the patient survived nearly 20 months after ECT. In the third patient, we observed disease progression 6 months after ECT; a second cycle of treatment was administered, with no response.

We did not observe any association between previous radiation and response rate $(P=0.38)$ or complete response rate $(P=1.0)$ to ECT. The response rate was $5 / 5(100 \%)$ and $12 / 14(86 \%)$ in patients with G2 and G3 angiosarcomas, respectively $(P=1.0)$; the CR rate was $4 / 4$ $(80 \%)$ versus $4 / 14(29 \%)$, respectively $(P=0.11)$

\section{Tumor Control}

Local failure (i.e., recurrence or progression within ECT field) occurred in four and two patients, respectively, after a median interval of 4.5 months (range, 2-9) (Fig. 4). Two of these six patients underwent a second ECT cycle: the first patient achieved a CR with durable $(>6$ months) local control, while the other proved unresponsive and developed further metastases shortly thereafter; salvage surgery was attempted with palliative intent. Five out of 19 patients $(26.3 \%)$ developed out-of-field progression, after a median interval of 5.9 months (range, 4.7-12.8). For this reason, two of these five patients received up to three ECT cycles with partial response and durable local control. After a median follow-up of 12 months (range, 3-48), 1-year 

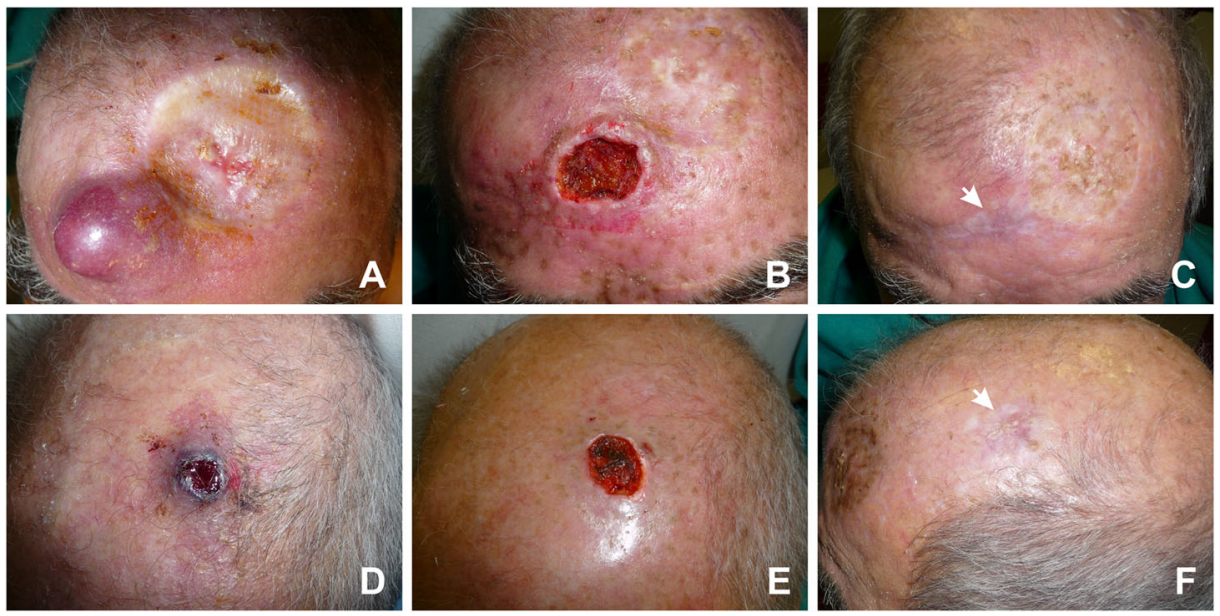

Fig. 1. Locally recurrent, multifocal angiosarcoma of the scalp. Baseline presentation of two target lesions treated by electrochemotherapy $(\mathbf{A}, \mathbf{D})$. Tumor shrinkage and residual skin ulcer at 1 month following treatment $(\mathbf{B}, \mathbf{E})$; wound healing and long lasting tumor response after 6 months $(\mathbf{C}, \mathbf{E})$.

LPFS was 68\% (95\%CI 41-94\%) (Supplemental Fig. S1). The local failure rate in patients who received only ECT was higher compared to patients who received further systemic treatment after ECT (5/12 (42\%) versus $1 / 7(14 \%)$, respectively, $P=0.33)$. At the last follow-up, the local response rate in patients $(n=13)$ who received ECT alone was comparable to patients $(\mathrm{n}=6)$ who received further oncological treatments, $9 / 13(69.2 \%)$ versus $4 / 6(66.7 \%)$, respectively $(P=0.91)$. One-year PFS in the whole cohort was $45 \%$ (95\%CI 12-69\%) (Supplemental Fig. S1).

\section{Toxicity}

No serious adverse events were reported during the procedure and hospital stay. Nine patients developed different grades of cutaneous toxicity: $\mathrm{G} 1$ ( $\mathrm{n}=6$ patients, $32 \%$, with erythema, tissue oedema and skin ulceration); $\mathrm{G} 2$ ( $\mathrm{n}=1$ patient, $5 \%$, with skin ulceration); and G3 ( $\mathrm{n}=2$ patients, $11 \%$, with skin ulceration requiring wound dressing for 4 and 6 months, respectively). Both patients who reported G3 ulceration had tumors located on the upper limb and a history of previous
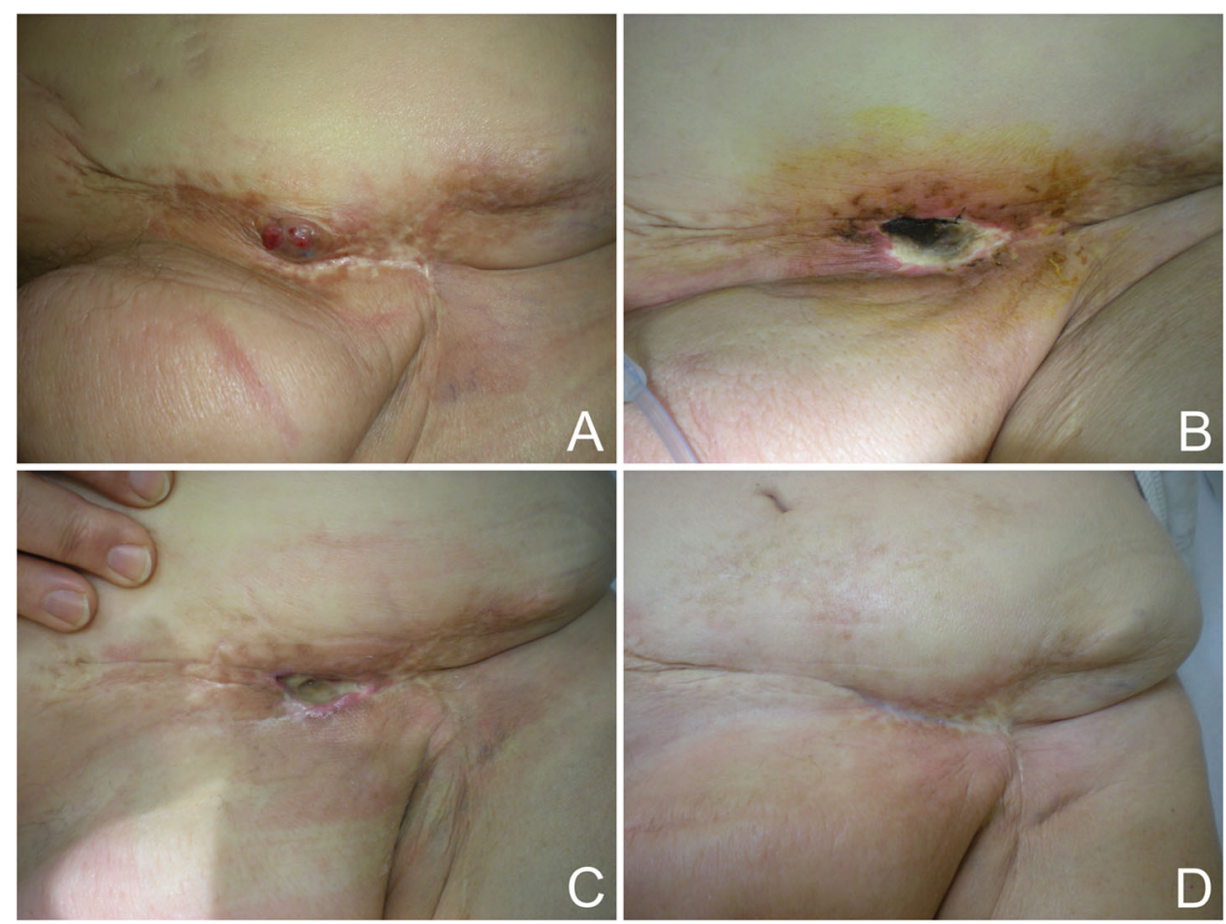

Fig. 2. Cutaneous angiosarcoma in a patient with previous penile squamous cell carcinoma treated with radiotherapy. Local recurrence on the abdominal wall after previous excision and radiation (A); necrotic tumor 15 days after ECT (B); complete regression after 2 months $(\mathbf{C})$; the patient was disease-free at 36 months (D). 

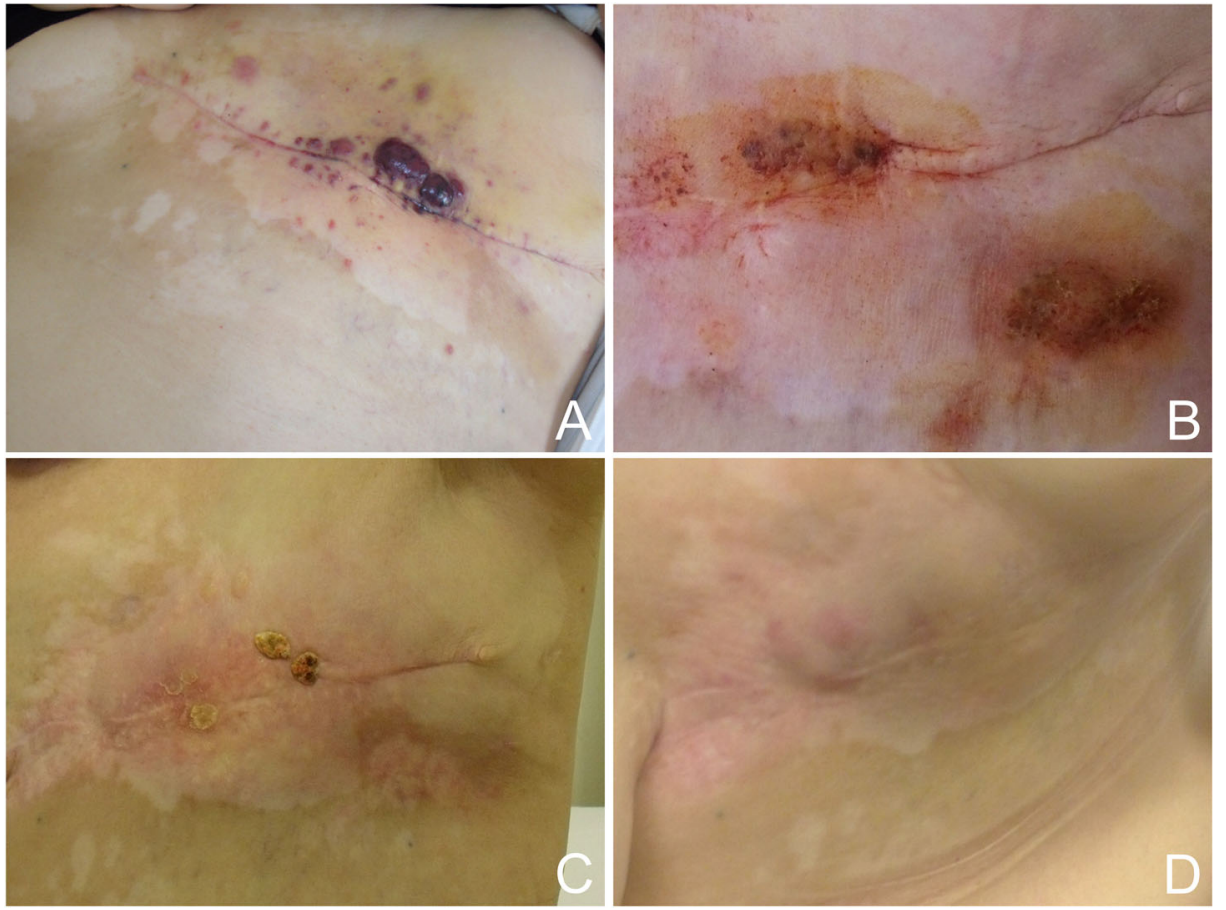

Fig. 3. Multifocal recurrence of radiation-induced mammary angiosarcoma. Skin metastases at the site of previous surgical excisions (A). Large scabs 1 month after ECT (B); Partial response after 2 months (C); the patient was local disease-free 8 months after ECT (D).

radiotherapy; they also received cytotoxic chemotherapy following ECT. There was a trend for more frequent and more severe toxicity in patients who were previously irradiated for angiosarcoma compared with those who were not ( $4 / 4$ vs. $5 / 10$, respectively, $P=0.03)$. In irradiated patient skin toxicity grade was G1, G2, G3, and G3; in nonirradiated patients, skin toxicity was G1 in all cases. All the toxicities resolved within 6 months.

In 10 patients $(52.6 \%)$, treated tumors were reported to be moderately to highly painful at the time of enrollment (Fig. 5). Two months after ECT, pain level was significantly reduced and was indicated as "moderate" by only four patients $(P=0.01)$. Out of 54 target lesions, 7 (13\%) were bleeding at baseline; at the last follow-up, in five of these seven tumors bleeding was controlled; intermittent bleeding was still reported by a single patient with two lesions. Overall, only one out of 6 patients with bleeding tumors at baseline did not have hemostasis at last follow-up.

\section{Patient Outcome}

Visceral metastases developed in 4 out of $19(21 \%)$ patients, after an interval of 6, 6, 8, and 14 months, respectively. At the last follow-up, patient status was as follows: disease-free, $n=4$; alive with local

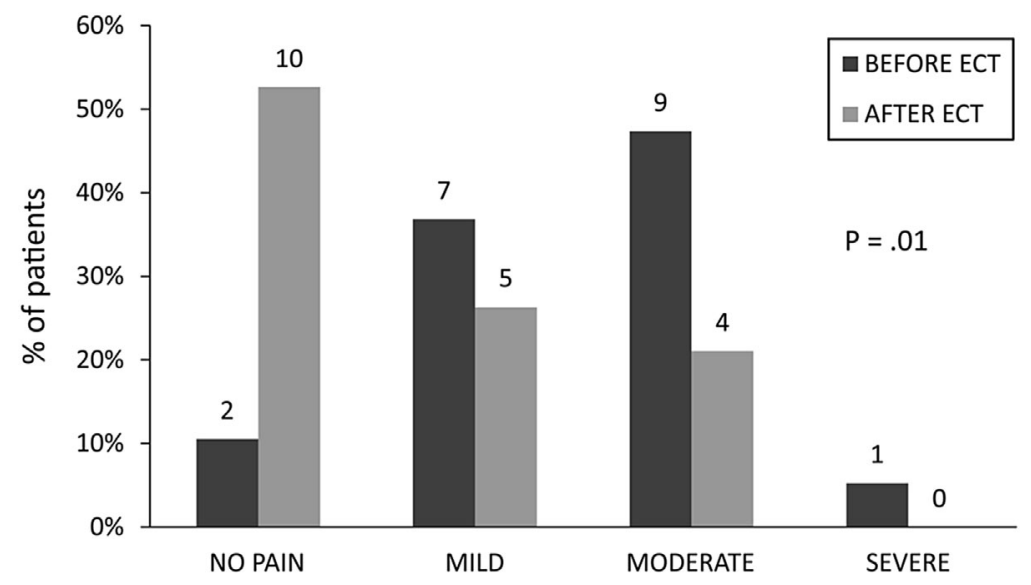

Fig. 4. Patient reported pain levels before and after ECT. Post-ECT assessment was performed at the first follow-up visit, after a median time of 29 days (range, 23-46). Pain intensity was graded by means of a 10-point Verbal Numerical Rating Scale (VNRS) as follows: 0, no pain; 1-2, mild pain; 3-6, moderate pain; 7-10, severe pain. 


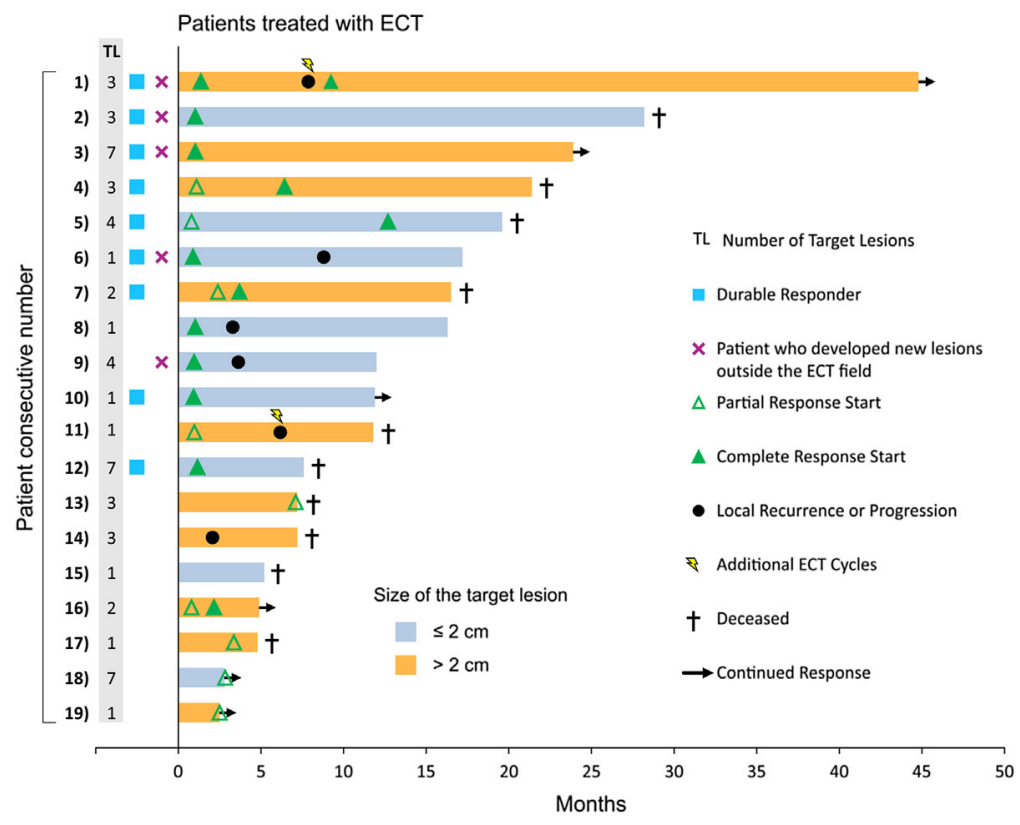

Fig. 5. Swimmer plot graph of time to and durability of local tumor response of baseline tumors. Each bar represents one patient. A durable responder (indicated by symbol on the left of the "y" axis) is a subject who had confirmed response for at least 6 months. A patient who experienced disease recurrence outside the ECT field is indicated by $\times$ symbol (additional ECT cycles applied on out-of-field recurrences are not shown). A patient who experienced local failure (disease progression or relapse) within ECT field is indicated by symbol on the bar. The bars $\mathrm{n}^{\circ}$ $2,6,11,14,17$ (starting from the top) correspond to the patients who were previously irradiated for angiosarcoma. The bars $n^{\circ} 2,5,11$ (starting from the top) correspond to the patients with angiosarcomas of the soft tissue. The bar $\mathrm{n}^{\circ} 14$ (starting from the top) indicate the single patient who was receiving concomitant treatment with paclitaxel at the time of ECT.

disease, $\mathrm{n}=5$; dead of disease, $\mathrm{n}=10$. Median OS after ECT of the entire cohort was 12 months (range, 4-44); OS was longer in patients with locally advanced compared with metastatic disease, 17 (range, $8-44)$ versus 8.8 (range, $4-16)$ months, respectively $(P=0.10)$. The median OS since the diagnosis of primary angiosarcoma was 29.9 months (range, 14.0-49.8).

\section{DISCUSSION}

This study examined, for the first time, outcomes associated with use of ECT in angiosarcoma patients. According to our results, ECT seems a promising treatment modality for providing effective local tumor control and symptom palliation in angiosarcoma patients. In fact, it ensured durable disease stabilization in 9 out 19 of our patients (Fig. 5), while causing relevant, although transient, toxicity in only two cases. Taking into account the number of local failures ( $\mathrm{n}=6$ patients) and the number of out-of-field relapses $(n=5$ patients), we can roughly estimate that a quarter of patients derived a clinical benefit from local treatment with ECT. In particular, these patients achieved durable $(\geq 6$ months) and effective tumor control, without experiencing relevant skin toxicity. These results may have implications for the management of angiosarcoma patients. In fact, since locally advanced/metastatic angiosarcoma has poor prognosis and treatment is considered palliative in most cases, tolerability of applied intervention is crucial.

Radical resection represents the mainstay of treatment, possibly in the frame of a multimodal approach [25,26]. Unfortunately, in everyday practice most patients are elderly and often inoperable or unfit for conventional oncological treatments. ECT is an emerging skin-directed therapy with proven efficacy across malignanciesmainly in melanoma [27], - and a favorable toxicity profile [28]. Before our analysis, it was unclear whether ECT with bleomycin had activity in angiosarcomas. Due to some intrinsic constraints (i.e., the length of the electrodes for electric pulse delivery), our series mainly accounts for patients with superficial tumors, such as cutaneous and breast angiosarcomas. Before ECT, most patients received surgery as the exclusive treatment for recurrence. It is conceivable that previous radiotherapy (12/19 patients) as well as patient age and associated morbidities could have prevented physicians from more aggressive treatment. Woefully, the median interval between surgical resection and subsequent local failure was 3.5 months, which is consistent with the time to local recurrence reported by Jallali et al. [11] in patients with radiation-induced angiosarcoma after a microscopically incomplete excision. It is conceivable that precocity of relapse in our patients could have been related to the high rate $(35 \%)$ of R1 resections and to the avoidance or impossibility to administer adjuvant therapies. A radical treatment is an established prognostic factor in angiosarcoma $[15,25,26]$, therefore the introduction of an effective therapy could be beneficial in patients who cannot receive radical resection. Despite the inherent limitations of a retrospective analysis based on small numbers, our data suggest that ECT with bleomycin has sustained activity in this tumor. In fact, more than $80 \%$ of electroporated tumors were responsive to the first cycle of treatment and up to $67 \%$ of them showed complete regression. As a result, a high percentage of patients were responsive to ECT. This favorable early outcome was achieved without concomitant systemic therapies and irrespective of previous radiotherapy. Notably, tumor shrinkage was precocious, after a median interval of 30 days following treatment. Noteworthy, tumor control was durable $(>6$ months) in about half of patients (Fig. 4). Our 68\% 1-year LPFS compares favorably with PFS indicated for currently available systemic agents, such as doxorubicin, paclitaxel and bevacizumab [29,30]. Notwithstanding, our data on local tumor control may have been influenced by the small sample and patient heterogeneity and should therefore be considered with caution. 
Interestingly, we observed a symptomatic benefit in five out six patients with bleeding tumors, an effect which was likely due to the strong ECT anti-vascular effect. Moreover, we also observed an improvement of tumor-associated pain figures (Fig. 5), which is a relatively common complaint of patients with superficial angiosarcoma [16]. Generally, treatment-induced pain is one of the most relevant concerns with ECT, although it is well tolerated in most patients [28]. Overall, we observed a tendency for more frequent and more severe dermatologic side effects in previously irradiated patients. G3 skin ulceration was reported in two patients, who were both previously irradiated; in these patients, wound healing may have also been hampered by subsequent chemotherapy. The possibility to efficiently control superficial metastases, coupled with the favorable toxicity profile and ease of administration, make thus ECT a reasonable option also in patients with visceral metastases.

Standard chemotherapeutic agents for metastatic angiosarcoma include doxorubicin, ifosfamide, and taxanes [29]. In recent years, the inhibition of the vascular endothelial growth factor pathway has emerged as a rational strategy [30]. However, response rates remain invariably low (doxorubicin, 30\%; paclitaxel, 18-62\%; bevacizumab, $17 \%)$. On the other hand, angiosarcoma rich vasculature and superficial localization allow for optimal exploitation of ECT anti-vascular effects $[19,20]$. In radiation-associated sarcomas, the association of re-irradiation and hyperthermia produced a response rate of $75 \%$, but with relevant toxicities [26]. In patients with scalp angiosarcoma, different strategies based on radiotherapy (total scalp irradiation with $\mathrm{X}$-rays and electrons, radiotherapy combined with interleukin-2 immunotherapy, and definitive radiotherapy combined with docetaxel) have been explored, unfortunately without definitive results [31-33]. Compared with these options, ECT has a number of advantages. It does not require dedicated facilities or specific expertise; it's a 1-day treatment that can be delivered in a day-hospital or outpatient setting; it can be repeated several times, the main limiting factor being the cumulative dose of bleomycin $\left(400,000 \mathrm{IU} / \mathrm{m}^{2}\right)$.

Nevertheless, clinicians should be aware that bleomycin limiting toxicity is exerted on the lung. In fact, this drug may induce severe toxicity that should not be underestimated, particularly in elderly patients or in those who were previously irradiated. For these reason, patient age, renal function and previous bleomycin exposure are crucial information in patient selection for ECT.

In our study, 6 out of 19 patients developed in-field recurrence $(n=4)$ or progression $(n=2)$. Two of them received a second ECT cycle, which ensured durable local remission in one patient (Fig. 5, patient \#1). Thanks to its antivascular effect, ECT also represents a valuable palliative tool for patients with bleeding tumors as well as a highly active treatment in patients with Kaposi sarcoma [21,22]. In order for ECT to be effective, two conditions must be met: (i) an adequate drug concentration at tumor site and (ii) an adequate coverage of the target area with electric voltage. For these reasons, most angiosarcomas represent an ideal target for percutaneous treatment with ECT.

In future, an intriguing aspect will be the investigation of ECT outcome in relation with the local immune infiltrate. According to a recent study, tumor-infiltrating lymphocytes (TILs), in particular $\mathrm{CD} 8+$, seem to be the most relevant immune cell population affecting the prognosis of cutaneous angiosarcoma patients [33]. Interestingly, ECT has been shown to elicit a local immune response in melanoma patients and TILs distribution (a brisk pattern) and their phenotype (CD8 expression) were associated with a higher probability of response to treatment [34]. Interestingly, according to Maddox et al. [8] although most angiosarcomas had a no-brisk lymphocytic infiltrate, those with a marked infiltrate showed a significantly longer interval to recurrence. There is no question that the present report has unavoidable weaknesses, due to small numbers, the retrospective fashion, as well as patient heterogeneity. Despite these limitations, however, our study indicates sustained response rate with ECT in a difficult-to-treat malignancy with limited therapeutic options. Moreover, our study involved centers that have long used ECT according to a shared protocol and that conduct studies with ECT also in other tumors $[35,36]$.

In conclusion, our results indicate that ECT exerts sustained antitumor activity in angiosarcomas. It ensured durable local control in about a half of patients in our study. In fact, 9/19 patients experienced durable local tumor control and five of them remained also free from out-of-field progression. Symptom benefits included bleeding control and local pain relief. Based on these favorable results, we envision that ECT could be further tested in appropriately designed trials. Further collaborative efforts will help to clarify the role of this treatment modality for improving local control and patient outcome in this challenging disease.

\section{ACKNOWLEDGMENTS}

Michela Battista for data collection; Francesca Deterlizzi for statistical analyses; Pierantonio Gallo and Renzo Grancara, Department of Surgery, Oncology and Gastroenterology of the University of Padova for informatics support; Associazione Onlus "Maria Ruggieri per la ricerca angiosarcoma e tumori rari" for supporting the study; Associazione Piccoli Punti Onlus, Padova, for supporting networking activities; Christina Drace, Veneto Institute of Oncology, for editing the manuscript.

\section{REFERENCES}

1. Young RJ, Brown NJ, Reed MW, et al.: Angiosarcoma. Lancet Oncol 2010;11:983-991.

2. Koch M, Nielsen GP, Yoon SS: Malignant tumors of blood vessels: Angiosarcomas, hemangioendotheliomas, and hemangioperictyomas. J Surg Oncol 2008;97:321-329.

3. Mark RJ, Poen JC, Tran LM, et al.: Angiosarcoma. A report of 67 patients and a review of the literature. Cancer 1996;77:2400-2406.

4. Fayette J, Martin E, Piperno-Neumann S, et al.: Angiosarcomas, a heterogeneous group of sarcomas with specific behavior depending on primary site: A retrospective study of 161 cases. Ann Oncol 2007;18:2030-2036.

5. Albores-Saavedra J, Schwartz AM, Henson DE, et al.: Cutaneous angiosarcoma. Analysis of 434 cases from the surveillance, epidemiology, and end results program, 1973-2007. Ann Diagn Pathol 2011;15:93-97.

6. Salas S, Stoeckle E, Collin F, et al.: Superficial soft tissue sarcomas (S-STS): A study of 367 patients from the French Sarcoma Group (FSG) database. Eur J Cancer 2009;45:2091-2102.

7. Rossi CR, Vecchiato A, Mastrangelo G, et al.: Adherence to treatment guidelines for primary sarcomas affects patient survival: A side study of the European CONnective TIssue CAncer NETwork (CONTICANET). Ann Oncol 2013;24:1685-1691.

8. Maddox JC, Evans HL: Angiosarcoma of skin and soft tissue: A study of forty-four cases. Cancer 1981;48:1907-1921.

9. Pawlik TM, Paulino AF, McGinn CJ, et al.: Cutaneous angiosarcoma of the scalp: A multidisciplinary approach. Cancer 2003;98:1716-1726.

10. Ogawa K, Takahashi K, Asato Y, et al.: Treatment and prognosis of angiosarcoma of the scalp and face: A retrospective analysis of 48 patients. Br J Radiol 2012;85:e1127-e1133.

11. Jallali N, James S, Searle A, et al.: Surgical management of radiation-induced angiosarcoma after breast conservation therapy. Am J Surg 2012;203:156-161.

12. Torres KE, Ravi V, Kin K, et al.: Long-term outcomes in patients with radiation-associated angiosarcomas of the breast following surgery and radiotherapy for breast cancer. Ann Surg Oncol 2013;20:1267-1274.

13. Morgan EA, Kozono DE, Wang Q, et al.: Cutaneous radiationassociated angiosarcoma of the breast: Poor prognosis in a rare secondary malignancy. Ann Surg Oncol 2012;19:3801-3808. 
14. Seinen JM, Styring E, Verstappen V, et al.: Radiation-associated angiosarcoma after breast cancer: High recurrence rate and poor survival despite surgical treatment with R0 resection. Ann Surg Oncol 2012;19:2700-2706.

15. Abraham JA, Hornicek FJ, Kaufman AM, et al.: Treatment and outcome of 82 patients with angiosarcoma. Ann Surg Oncol 2007;14:1953-1967.

16. Penel N, Marreaud S, Robin YM, et al.: Angiosarcoma: State of the art and perspectives. Crit Rev Oncol Hematol 2011;80:257-263.

17. Ducimetiere F, Lurkin A, Ranchere-Vince D, et al.: Incidence of sarcoma histotypes and molecular subtypes in a prospective epidemiological study with central pathology review and molecular testing. PLoS ONE 2011;6:e20294.

18. Mir LM, Orlowski S: Mechanisms of electrochemotherapy. Adv Drug Deliv Rev 1999;35:107-118.

19. Sersa G, Jarm T, Kotnik T, et al.: Vascular disrupting action of electroporation and electrochemotherapy with bleomycin in murine sarcoma. Br J Cancer 2008;98:388-398.

20. Jarm T, Cemazar M, Miklavcic D, et al.: Antivascular effects of electrochemotherapy: Implications in treatment of bleeding metastases. Expert Rev Anticancer Ther 2010;10:729-746.

21. Gehl J, Geertsen PF: Efficient palliation of haemorrhaging malignant melanoma skin metastases by electrochemotherapy. Melanoma Res 2000;10:585-589.

22. Curatolo P, Quaglino P, Marenco F, et al.: Electrochemotherapy in the treatment of Kaposi sarcoma cutaneous lesions: A two-center prospective phase II trial. Ann Surg Oncol 2012;19:192-198.

23. Mir LM, GJ, Sersa G, et al.: Standard operating procedures of electrochemotherapy: Instructions for the use of bleomycin or cisplatin administered either systemically or locally and electric pulses delivered by means of invasive or non-invasive electrodes. Eur J Cancer Suppl 2006;4:14-25.

24. Eisenhauer EA, Therasse P, Bogaerts J, et al.: New response evaluation criteria in solid tumours: Revised RECIST guideline (version 1.1). Eur J Cancer 2009;45:228-247.

25. Lahat G, Dhuka AR, Lahat S, et al.: Outcome of locally recurrent and metastatic angiosarcoma. Ann Surg Oncol 2009;16:2502-2509.

26. de Jong MA, Oldenborg S, Oei Bing, et al.: Reirradiation and hyperthermia for radiation-associated sarcoma. Cancer 2012;118:180-187.
27. Campana LG, Testori A, Mozzillo N, et al.: Treatment of metastatic melanoma with electrochemotherapy. J Surg Oncol 2014;109: 301-307.

28. Quaglino P, Matthiessen LW, Curatolo P, et al.: Predicting patients at risk for pain associated with electrochemotherapy. Acta Oncol 2015;54:298-306.

29. Italiano A, Cioffi A, Penel N, et al.: Comparison of doxorubicin and weekly paclitaxel efficacy in metastatic angiosarcomas. Cancer 2012;118:3330-3336.

30. Agulnik M, Yarber JL, Okuno SH, et al.: An open-label, multicenter, phase II study of bevacizumab for the treatment of angiosarcoma and epithelioid hemangioendotheliomas. Ann Oncol 2013;24:257-263.

31. Hata M, Wada H, Ogino I, et al.: Radiation therapy for angiosarcoma of the scalp: Treatment outcomes of total scalp irradiation with X-rays and electrons. Strahlenther Onkol 2014;90: 899-904.

32. Ohguri T, Imada H, Nomoto S, et al.: Angiosarcoma of the scalp treated with curative radiotherapy plus recombinant interleukin-2 immunotherapy. Int J Radiat Oncol Biol Phys 2005;61:1446-1453.

33. Miki Y, Tada T, Kamo R, et al.: Single institutional experience of the treatment of angiosarcoma of the face and scalp. Br J Radiol 2013;86:20130439.

34. Fujii H, Arakawa A, Utsumi D, et al.: $\mathrm{CD}^{+}$tumor-infiltrating lymphocytes at primary sites as a possible prognostic factor of cutaneous angiosarcoma. Int J Cancer 2014;34:2393-2402.

35. Quaglino P, Osella-Abate S, Marenco F, et al.: FoxP3 expression on melanoma cells is related to early visceral spreading in melanoma patients treated by electrochemotherapy. Pigment Cell Melanoma Res 2011;24:734-736.

36. Cabula C, Campana LG, Grilz G, et al.: Electrochemotherapy in the treatment of cutaneous metastases from Breast cancer: A multicenter cohort analysis. Ann Surg Oncol 2015;3:442-450.

\section{SUPPORTING INFORMATION}

Additional supporting information may be found in the online version of this article at the publisher's web-site. 